\title{
Novel Method for Predicting Limit Performance of Bus-Transfer Switching by Disconnectors
}

\section{Journal Article}

Author(s):

Ritter, Andreas (D); Straumann, Ueli; Franck, Christian (1)

Publication date:

2017-10

Permanent link:

https://doi.org/10.3929/ethz-b-000121962

Rights / license:

In Copyright - Non-Commercial Use Permitted

Originally published in:

IEEE Transactions on Power Delivery 32(5), https://doi.org/10.1109/TPWRD.2016.2609682 


\title{
Novel Method for Predicting Limit Performance of Bus-Transfer Switching by Disconnectors
}

\author{
Andreas Ritter, Student Member, IEEE, Ueli Straumann, Senior Member, IEEE, \\ and Christian M. Franck, Senior Member, IEEE
}

\begin{abstract}
In order to accurately predict the performance of disconnectors conducting bus-transfer switching in gas insulated substations (GIS), the influencing factors of the switching process itself as well as the reignition characteristics need to be analyzed. For this, an LC-oscillating AC current source in combination with a non-standardized full bus-transfer loop was built. Based on experiment data from a total of 347 reignitions and 570 extinctions, the reignition characteristic of the disconnector at current zero was investigated. Thereby, the derivation of a reignition criterion depending exclusively on the prediction of the instantaneous recovery voltage and the prospective arc voltage was possible. Using this criterion, time-dependent simulations of the bus-transfer process can be made to predict the performance of disconnectors for a wide variety of scenarios such as worst-case bus-transfer current for varying circuit parameters and ratings. Thus minimizing the need for extensive testing during substation planning or disconnector development.
\end{abstract}

Index Terms-disconnector, bus-transfer, current-interruption, gas insulated substations, testing, iec

\section{INTRODUCTION}

D ISCONNECTORS are an important part in high voltage switchgear installations. Their typical usage is the combination with circuit breakers, where disconnectors provide an isolation distance (coming along with increased requirement in regard of withstand voltages) as well as the ability of making and breaking of small capacitive currents (by energizing and de-energizing parts of the substations, such as busbars, bushings, current and voltage transformers).

In some substation topologies, such as the double-busbararrangements, bus-transfer current switching is also required. Due to the lower surge impedances of gas insulated metalenclosed switchgear (GIS) in comparison to their corresponding air insulated variants (AIS), the bus-transfer ratings are typically lower in GIS-applications (e.g. IEC 62271-102 [1]), even though, those ratings are currently under revision. When it comes to testing, the usual choice of test-setups are "oneloop" arrangements powered by a high current voltage source, see IEC 62271-102 [1]. An equivalent alternative to this, are full-loop setups [2]. By employing a current source to supply two parallel impedances, these setups provide a high degree of freedom in adjusting the pre-switching current as well as the recovery voltage. Especially when matching the resistance and inductance per unit length of the desired substation with the

A. Ritter and C. M. Franck are with the Power Systems and High Laboratories (EEH), ETH Zurich, Zurich 8092, Switzerland (e-mail: rittera@ethz.ch).

U. Straumann is with ABB Switzerland Ltd.

This work was financially supported by ABB Switzerland Ltd.

Manuscript received ?; revised ? setup's impedances, low-frequency high-current applications can be investigated in laboratory settings. Thus minimizing the need for full-scale GIS testing.

In an effort to study the arcing and reignition properties of a standard disconnector built for use in $420 \mathrm{kV}$ GIS, extensive testing using such a full bus-transfer setup was conducted. Pre-switching currents and recovery voltages within, as well as outside, of the range of capabilities specified by the current IEC standard were utilized. A focus was placed on analyzing the current and electrode separation distance dependencies of the arc voltage in order to model the time of current zero during the switching process. To predict reignition after current zero, the connection between arcing current and reignition voltage was studied, leading to a criterion which can be used to simulate worst-case scenarios for bus-transfer process in similar disconnectors while varying the crucial parameters such as pre-switching current, loop length and electrode separation speed.

\section{Bus-Transfer Setup}

To gain a deeper understanding of the interaction between recovery voltage and arc voltage during bus-transfer switching by GIS disconnectors, a test setup according to the equivalent circuit diagram in Fig. 1 was constructed. The main components of this setup consist of the LC-oscillating current source $\left(L_{\mathrm{S}}, C_{\mathrm{S}}, R_{\mathrm{S}}\right)$ and the full bus-transfer loop $\left(L_{1}, R_{1}, L_{2}, R_{2}\right.$, disconnector). Prior publication [2] elucidates the differences between this setup and the standardized bus-transfer test according to IEC 62271-102 [1]: With an initial capacitor voltage between $0-15 \mathrm{kV}$ the LC-oscillating current source can drive currents up to $4.5 \mathrm{kA}$ at $55 \mathrm{~Hz}$ between the source inductance $L_{\mathrm{S}}$ of $9.2 \mathrm{mH}$ and the capacitor bank $C_{\mathrm{S}}$ of $923 \mu \mathrm{F}$. Due to the exponential damping of the current through the ohmic resistance $R_{\mathrm{S}}$, the source current $i_{\mathrm{S}}$ decreases by $13 \%$ during one half-wave. $R_{\mathrm{S}}$ is itself not a discrete component but represents the sum of all ohmic losses in the source of which the windings of $L_{\mathrm{S}}$ contribute most. The flow of the source current $i_{\mathrm{S}}$ is initiated and interrupted by a medium-voltage vacuum circuit-breaker, selected for its low ohmic losses in closed state.

The full bus-transfer loop is composed of two identical impedances, one forming $R_{1}$ and $L_{1}$, the second forming $R_{2}$ and $L_{2}$. As explained in [2] these two impedances are specifically built to be able to imitate varying lengths of GIS busbar with respect to inductance and resistance. Fig. 2 shows equivalent length of GIS busbar in terms of resistance 
and inductance as a function of the number of turns of the impedance which are in use. The nearly linear increase in inductance per turn is achieved by winding low ohmic conductors on coils of sufficiently large diameter and pitch. It is evident, that with the available number of turns, busbar loops ranging from only a few meters up to $600 \mathrm{~m}$ can be emulated which satisfies all of the GIS substation arrangements found in the recent survey of Cigré Working Group A3.28 [3]. The unit under test is a GIS disconnector designed for a rated voltage of $420 \mathrm{kV}$ and operated at the rated filling pressure of $0.5 \mathrm{MPa}$ of $\mathrm{SF}_{6}$. This particular model features annular tungsten-copper $(80 \% \mathrm{Cu}, 20 \% \mathrm{~W})$ arcing electrodes with a contact area of $60 \mathrm{~cm}^{2}$ which are separated at a speed of $100 \mathrm{~mm} / \mathrm{s}$ without any active arc cooling mechanisms.

A large number of experiments was performed in this arrangement, focussing on studying the influences of individual factors independent of each other. The main considerations were towards varying pre-switching current and recovery voltage. By means of an extensive analysis toolbox, the raw measurement data of $u_{\mathrm{C}}, i_{\mathrm{S}}, i_{2}$ and $u_{\mathrm{DS}}$ were compensated for unavoidable measurement errors such as inductive coupling between the high current paths and the measurement cables. The main objective of the analysis toolbox as presented by the authors in [4], however, was to provide methods to extract the maximum information possible about the bus-transfer process. Automatic calculation of the recovery voltage at every point in time as well as averaging of arcing voltages was central to the evaluation of individual bus-transfer measurements.

\section{Arc Voltage Measurements}

Over the course of the evaluation, 570 bus-transfers were performed using the same disconnector but at varying source and bus-transfer loop parameters. The majority of said measurements exhibited extinction of the switching arc at the first current zero. However, through increasing pre-switching current and parallel impedance $L_{1}$ and $R_{1}$ multiple reignitions were made possible leading to 347 recorded reignitions at current zero and therefore a total of 917 recorded current zeros. A

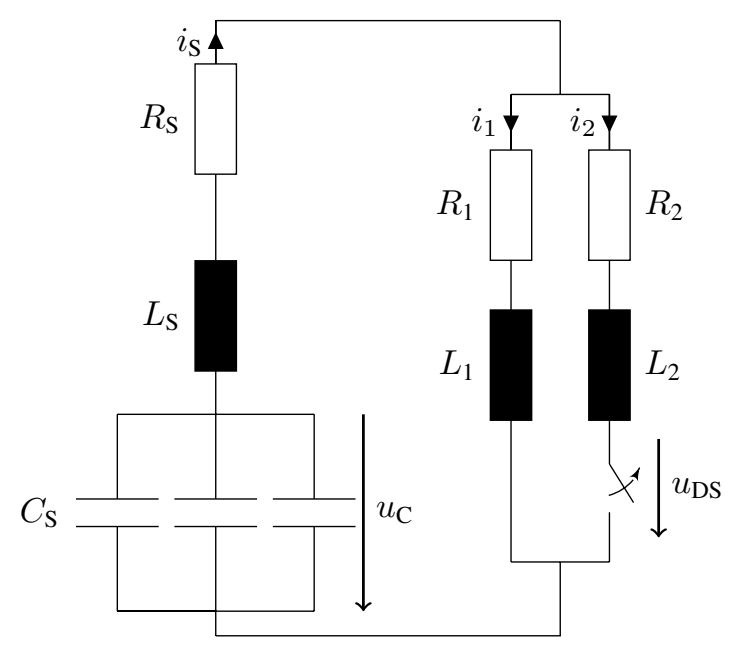

Fig. 1. Equivalent circuit diagram of the LC-oscillating current source connected to the full bus-transfer loop.

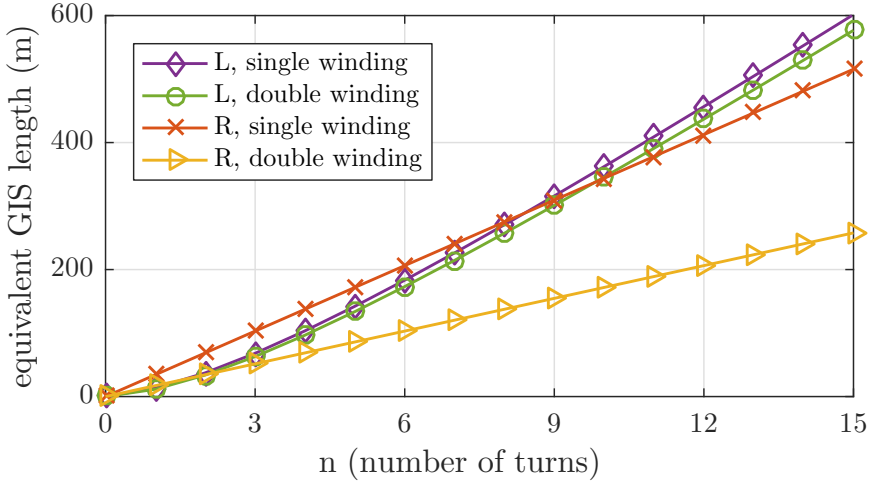

Fig. 2. Properties of the purpose-built bus-transfer coils: Inductance for single and double windings as well as resistance using single or double windings in terms of their equivalent length of GIS bus.

typical measurement exhibiting multiple reignitions is shown in Fig. 3. The depicted example shows the ignition of the initial switching arc virtually at the beginning of the first half wave of the source current, however, this ignition angle was varied at random over similar measurements. After the initial arc ends at the first current zero a subsequent arc of opposite voltage sign ignites instantly. The same occurs another four times until after the sixth current zero no further reignition takes place. The arc voltage plotted for this example displays six discrete, increasing levels which appear to be largely independent of the arc current. This is in agreement with the measurements performed by Yokomizu for copper-tungsten electrodes at similar distances in $\mathrm{SF}_{6}$ which showed a minimal ignition and extinction peak of a few Volts [5]. Due to the small changes in electrode separation distance between (re-)ignition and subsequent current zero, each voltage step was therefore approximated as constant for the analysis. In Fig. 4 each of the 917 arc voltage steps is given with respect to the absolute

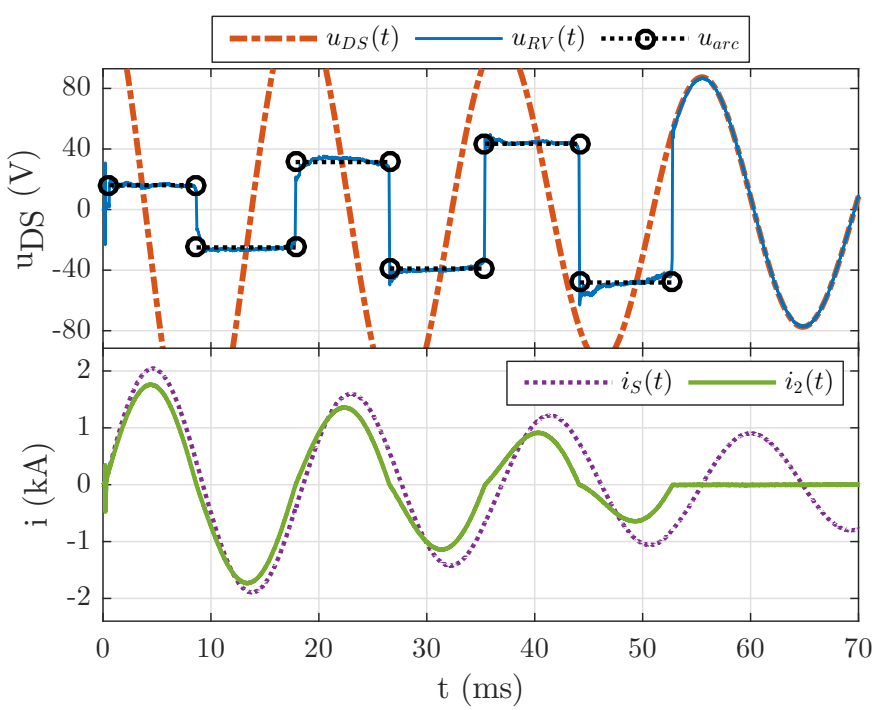

Fig. 3. Example measurement results exhibiting five reignitions. Top: measured disconnector voltage $u_{\mathrm{DS}}$ overlaid with simulated recovery voltage $u_{\mathrm{RV}}$ and approximated arc voltages; Bottom: measured currents through the disconnector $i_{2}$ and from the source $i_{S}$. 
voltage and its median time between ignition and current zero. Due to the known and very constant separation speed of the electrodes, the time since first arc ignition is readily convertible to electrode distance. As expected from measurements at similarly small electrode distances by Yokomizu [5] and van Engel [6] the overall development of arc voltages follows a square-root shape. The somewhat steeper increase in arc voltage with respect to distance compared to Yokomizu's results with similar electrode materials can be attributed to the higher gas pressure giving rise to increased cooling power of the arc column and thus higher arc voltages. As examples, the minimum arc voltage at $1 \mathrm{~mm}$ electrode separation distance in Fig. 4 is $23 \mathrm{~V}$ which is comparable to Yokomizu's $24 \mathrm{~V}$ at the same distance. However, at a separation distance of $3 \mathrm{~mm}$ Yokomizu's result of $30 \mathrm{~V}$ is significantly lower than the $37 \mathrm{~V}$ shown in Fig. 4. The spread of measured arc voltages for any given arcing time can be traced to three main sources. The primary source is the stochastic movement of the arc roots on the electrodes temporarily leading to an arc length which is higher than the electrode distance. A secondary source is the small error introduced by approximating the individual arcs with constant voltage to facilitate the data acquisition. Furthermore, the spread of arc voltages at arcing time zero largely coincides with the range of electrode drops found for pure tungsten and tungsten-copper in $\mathrm{SF}_{6}(13.8 \mathrm{~V}$, resp. $17.5 \mathrm{~V} \mathrm{[5])} \mathrm{indicating} \mathrm{some} \mathrm{erosion} \mathrm{of} \mathrm{the} \mathrm{electrode} \mathrm{material}$ over the course of the measurement series.

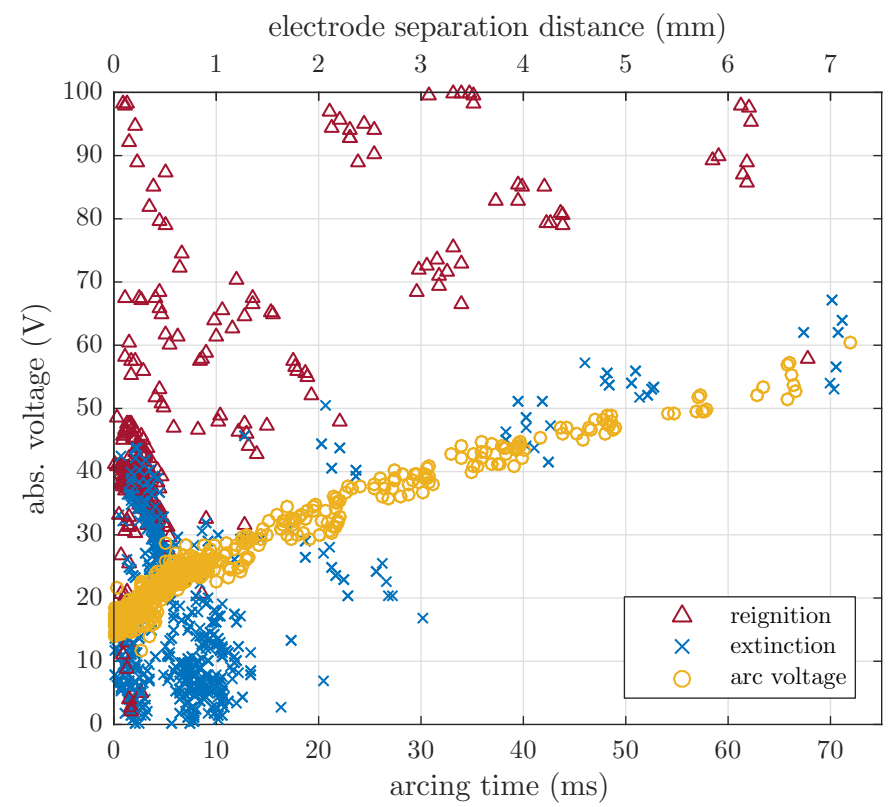

Fig. 4. Averaged arc voltages compared to recovery voltages at reignition and extinction, all voltages are given as absolute values and independent of the switching current.

\section{InFLUENCE of Instantaneous ReCOVERy Voltage}

Unlike the arc voltage which depends on properties of the disconnector under test, the instantaneous recovery voltage $u_{\mathrm{RV}}$ is determined solely by circuit parameters. After extinction of the switching arc, the entire source current $i_{\mathrm{S}}$ is conducted through the path parallel to the disconnector thus leading to the recovery voltage as given in equation (1) which can be measured by the same voltage sensors as the arc voltage. Due to high damping and low parasitic capacitances of the test circuit, no significant transient recovery is measurable as seen in Fig. 3.

$$
u_{\mathrm{RV}}(t)=i_{\mathrm{S}}(t) \mathrm{R}_{1}+\frac{d i_{\mathrm{S}}(t)}{d t} \mathbf{L}_{1}
$$

However, during periods of arcing the recovery voltage is inherently unmeasurable and thus has to be calculated. By calculating all circuit parameters from preceding open-circuit and short-circuit measurements, the aforementioned analysis toolbox is able to predict $u_{\mathrm{RV}}$ at any point in time during a bus-transfer. In Fig. 3 the prospective recovery voltage during and after bus-transfer is shown for an example measurement. It is evident that the simulated recovery voltage matches very accurately the measured disconnector voltage after extinction of the last switching arc. To analyze the influence of the recovery voltage on reignition of the switching arc, the prospective instantaneous recovery voltage at each current zero has been marked in Fig. 4. The main differentiation between the points is extinction of the arc (blue crosses) and reignition, i.e. development of a subsequent switching arc (red triangles). It is clear from the figure that the vast majority of switching arcs extinguishes if the recovery voltage at current zero is lower than the arc voltage of a subsequent arc would be. One individual example of this can be found in Fig. 3 which exhibits five reignitions at instantaneous recovery voltages above the arc voltage and a final extinction at a recovery voltage only a few Volts below the expected arc voltage. As is evident from the voltages of extinctions at small arcing times, however, the opposite is not always true. Especially in the first few milliseconds extinction is possible at recovery voltages more than twice the expected arc voltage in some cases. The exception to the reignition observation are current zeros at very small arcing times, i.e. $5 \mathrm{~ms}$ or less. At these

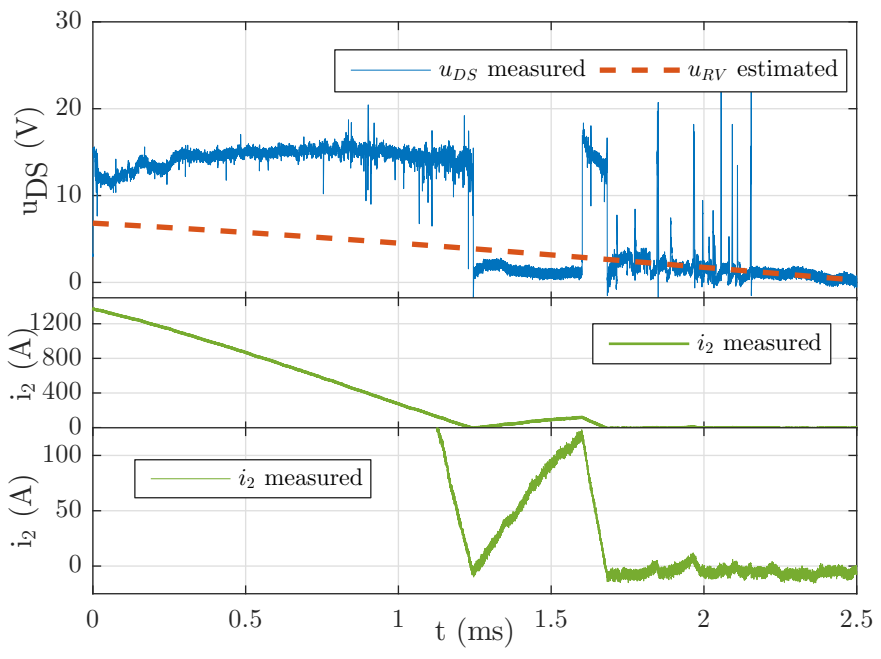

Fig. 5. Measured disconnector voltage $u_{\mathrm{DS}}$ and calculated recovery voltage $u_{\mathrm{RV}}$ (top), compared to disconnector current $i_{2}$ (middle and bottom); Extended period of current conduction from $1.2 \mathrm{~ms}$ to $1.7 \mathrm{~ms}$ after initial current zero followed by short arc and voltage flicker. 
small electrode distances, current flow at voltages below the minimum arc voltage (i.e. electrode fall voltage) is possible after the first current zero for brief times. Fig. 5 illustrates this behavior for the most extreme case recorded. At $1.25 \mathrm{~ms}$ the first current zero is reached, immediately afterward the current $i_{2}$ increases significantly while the disconnector voltage stays at levels of $1-2 \mathrm{~V}$, which are below the prospective recovery voltage. $0.35 \mathrm{~ms}$ later a brief arc forces the current back to zero again. The same process is repeated at significantly higher speeds a number of times until at $2.2 \mathrm{~ms}$ when the disconnector voltage stays equivalent to the simulated recovery voltage. Since this phenomenon only appears at very small electrode distances of less than $0.5 \mathrm{~mm}$ the likely cause is the appearance of molten metal bridges due to their similar timevoltage patterns during formation and subsequent rupture [7], [8]. At electrode distances above $0.5 \mathrm{~mm}$ (corresponding to arcing times above $5 \mathrm{~ms}$ ) such re-ignitions have not been measured, hence the underlying phenomena are assumed to disappear at such distances.

\section{Hypothesis of CONNECTION BETWEen ARCING AND RECOVERY VOLTAGES}

Any reignition after an arcing time of $5 \mathrm{~ms}$ is only observed if the prospective recovery voltage is higher than the expected arcing voltage as seen in Fig. 4. Since these reignitions occur in a nearly homogeneous electric field ${ }^{1}$ at voltages well below the Paschen curve for $\mathrm{SF}_{6}$ [9] without any forced gas flow to aid recovery of the electrode gap, all breakdowns must occur in the thermal phase after current zero as defined by Nakanishi [10]. Even though, there are indications that in similar free-burning arcs in air, the plasma is dominated by metallic vapor [11], with different dielectric properties than $\mathrm{SF}_{6}$ and its dissociation products. Albeit, contradicting results are presented by Yokomizu [12] showing domination of $\mathrm{SF}_{6}$ in arcs in similar geometries. Nonetheless, ionization by collision is expected to require voltages of similar magnitude in metallic vapour dominated situations also. The lack of recovery voltage measured between current zero and subsequent arc further substantiates this conclusion due to the small times in the microsecond range typical for thermal breakdowns. Unlike the longer timescales typical for dielectric reignition, phenomena in the range of microseconds are inherently unmeasurable by the test circuit. The high post arc current necessary for thermal breakdown also provides a substantial damping to the circuit, thus explaining the lack of transient recovery voltage after the last current zero.

The current source was built to have minimal interaction with the full bus-transfer loop by using a source inductance $L_{\mathrm{S}}$ significantly larger than the bus-transfer inductances $L_{1}$ and $L_{2}$. Therefore, the supply of current from the source was only marginally affected by the state of the bus-transfer process. When assuming that the conditions for thermal breakdown are given at each current zero, the reignition criterion thus becomes entirely dependent on the circuit. Hence, the current

\footnotetext{
${ }^{1}$ Due to the small electrode distance compared to the electrode surface, the electric field between the electrodes can be approximated as homogenous for all possible locations of arc roots.
}

$i_{2}$ flowing through the disconnector is a superposition of a source current part $i_{2}^{\mathrm{S}}$ and a bus-transfer part $i_{2}^{\text {arc }}$ as introduced in [4]. The source current $i_{\mathrm{S}}$ is given as

$$
i_{\mathrm{S}}(t)=\beta e^{-\delta t} \sin \omega_{s} t
$$

where variables $\beta, \delta$ and $\omega_{s}$ depend only on source parameters as shown in Fig. 1 in addition to the initial capacitor charge. Based in majority on the configuration of the bus-transfer loop, $i_{\mathrm{S}}$ is split into $i_{1}^{\mathrm{S}}$ and $i_{2}^{\mathrm{S}}$ where the latter is composed of a sinusoidal term and an exponentially decaying circulating current as given by

$$
i_{2}^{\mathrm{S}}(t)=\gamma e^{-\delta t} \sin \left(\omega_{s} t+\varphi\right)-\gamma \sin \varphi \cdot e^{-\frac{t}{\tau}} .
$$

The amplitude $\gamma$ as well as the phase angle $\varphi$ depend on both source and bus-transfer loop parameters whereas the time constant $\tau$ only depends on the sum of bus-transfer loop inductance and resistance according to

$$
\tau=\frac{L_{1}+L_{2}}{R_{1}+R_{2}}
$$

because $e^{\frac{-t}{\tau}}$ constitutes the homogeneous solution of the differential equations for the circulating current in the bustransfer loop.

In an ideal configuration of the bus-transfer loop, the fractions $L_{1} / R_{1}$ and $L_{2} / R_{2}$ are equal as they are defined by the type of substation that is modelled. In this case, the phase angle $\varphi$ becomes zero and $i_{2}^{\mathrm{S}}(t)$ simplifies to

$$
i_{2}^{\mathrm{s}}(t)=\frac{d_{1}}{d_{1}+d_{2}} \beta e^{-\delta t} \sin \omega_{s} t,
$$

where $d_{1}$ and $d_{2}$ are the lengths of the bus bars emulated according to Fig. 2. The loop current $i_{2}^{\text {arc }}$ caused by the switching arc can be expressed as

$$
i_{2}^{\operatorname{arc}}(t)=-\frac{u_{\operatorname{arc}}}{R_{1}+R_{2}}\left(1-e^{-\frac{\left(t-t_{i}\right)}{\tau}}\right)
$$

assuming a constant arc voltage $u_{\text {arc }}$ and an ignition time $t_{i}$. It is important to note that the sign of the arc voltage must be identical to the sign of the current $i_{2}$ through the disconnector as the arc is itself powered by this current.

From this relationship the connection between recovery voltage and arc voltage observed in the measurement results can be derived as follows. Assuming instant thermal breakdown upon current flow, immediately after the time of current zero $t_{0}$, the current through the disconnector becomes

$$
i_{2}(t)=i_{2}^{\mathrm{S}}(t)-i_{2}^{\mathrm{S}}\left(t_{0}\right) \cdot e^{-\frac{\left(t-t_{0}\right)}{\tau}}+i_{2}^{\operatorname{arc}}(t),
$$

where the second term compensates for the fact that $i_{2}$ generally does not reach current zero at the same time as $i_{\mathrm{S}}$ does. In order for $i_{2}(t)$ to increase in magnitude after $t_{0}$, the source terms have to grow faster than $i_{2}^{\text {arc }}(t)$. At time $t_{0}^{+}$ immediately after current zero, the time derivative of (7) can thus be rewritten to reflect this as

$$
\left.\frac{d i_{2}^{\mathrm{S}}(t)}{d t}\right|_{t=t_{0}^{+}}-\frac{1}{\tau} i_{2}^{\mathrm{S}}\left(t_{0}^{+}\right)>\frac{u_{\mathrm{arc}}}{L_{1}+L_{2}},
$$

which can be simplified to

$$
\left.\left(L_{1}+L_{2}\right) \cdot \frac{d i_{2}^{\mathrm{S}}(t)}{d t}\right|_{t=t_{0}^{+}}-\left(R_{1}+R_{2}\right) \cdot i_{2}^{\mathrm{S}}\left(t_{0}^{+}\right)>u_{\mathrm{arc}}
$$



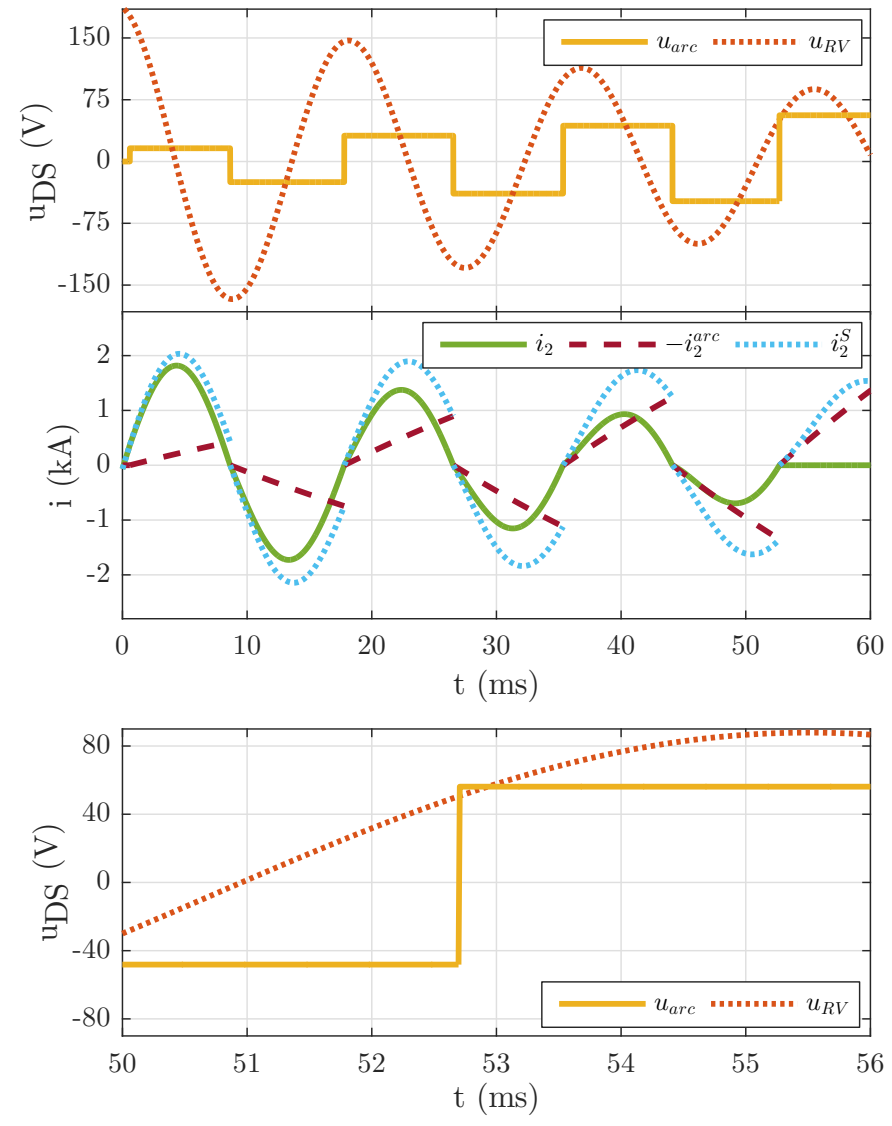

Fig. 6. Simulation of the measurement shown in Fig 3. Top: recovery voltage $u_{\mathrm{RV}}$ and arc voltages; Middle: disconnector current $i_{2}$ and its parts $i_{2}^{\mathrm{S}}(t)-$ $i_{2}^{\mathrm{S}}\left(t_{0}\right) \cdot e^{-\frac{\left(t-t_{0}\right)}{\tau}}$ (labelled $i_{2}^{S}$ ) as well as $-i_{2}^{\operatorname{arc}}(t)$. Bottom: Magnification of top plot around time of arc extinction.

As per definition of the recovery voltage in (1), the left side of (9) is equivalent to $u_{\mathrm{RV}}(t)$, thus finally leading to

$$
u_{\mathrm{RV}}\left(t_{0}\right)>u_{\mathrm{arc}}
$$

as the criterion for an increasing disconnector current magnitude after current zero. A visual interpretation of this criterion is shown in Fig. 6 which illustrates the development of the individual parts of (7) on the measurement presented as an example in Fig. 3. Immediately after each of the first five current zeros, the instantaneous recovery voltage is higher than the arc voltage which validates inequation (8). In the figure, this is represented by a faster increase of the source terms as dotted blue curve compared to the dashed purple curve which represents the negative current created by the switching arc. At the sixth current zero, the expected arc voltage is $10 \%$ higher than the instantaneous recovery voltage, leading to violation of (8). Therefore, the circuit cannot sustain an arc at this point leading to an interruption of the disconnector current. It is important to note, that less than $1 \mathrm{~ms}$ after the interruption, the criterion of (10) is fulfilled again, however, the conditions for an immediate reignitions have ceased to exist and no reignition occurs. It could, therefore, be assumed that the conditions necessary for thermal breakdown only persist for short times in the range of tens of microseconds after current zero which is in line with [10].
As can be seen in Fig. 4, however, the criterion of (10) is necessary but not sufficient for reignition. There are numerous interruptions at current zeros followed by recovery voltages significantly higher than the anticipated arcing voltages. The main reason for this discrepancy can be assumed to lie in the stochastic nature of the arc development. The measurement shown in Fig. 3 provides an example of this by exhibiting three reignitions at arc voltages above the averaged voltage. The approximation of $u_{\text {arc }}$ as constant between two current zeros is sensible to enable evaluation of large sets of data but neglects this stochastic behavior and any arc length dependency between two current zeros. Therefore, the criterion of (10) can be interpreted as a lower limit and thus be used to calculate worst-case bus-transfer switching processes. Additionally, the inverse of (10) stays true even when taking into account stochastic arc processes since these processes always lead to an arc voltage above the expected minimal voltage. As per the given derivation, no arc can occur if the recovery voltage immediately after current zero is below the expected minimal arc voltage with the exception of the previously explained extremely short arcing times below $5 \mathrm{~ms}$.

\section{IMPLICATIONS FOR DETERMINING LIMIT PERFORMANCE OF DISCONNECTORS}

By using the criterion of (10) as a worst-case criterion for reignition at each current zero, only a small number of circuit parameters in combination with a measured arc voltage behavior are needed to predict bus-transfer processes simulatively. The application of this prediction is, of course, not limited to the presented test setup but can be extended to apply to other types of tests as well as real-world applications as long as the operating principle of the device under test is the same as the evaluated disconnector.

One such example is the influence of the time of contact opening with respect to the pre-switching current as plotted in Fig. 7. In this figure, an arc voltage function over time similar to the one from Fig. 4 is assumed in combination with a current distribution and recovery voltage development according to a GIS substation with two parallel buses of $40 \mathrm{~m}$ (in series with the disconnector) and $160 \mathrm{~m}$. The material

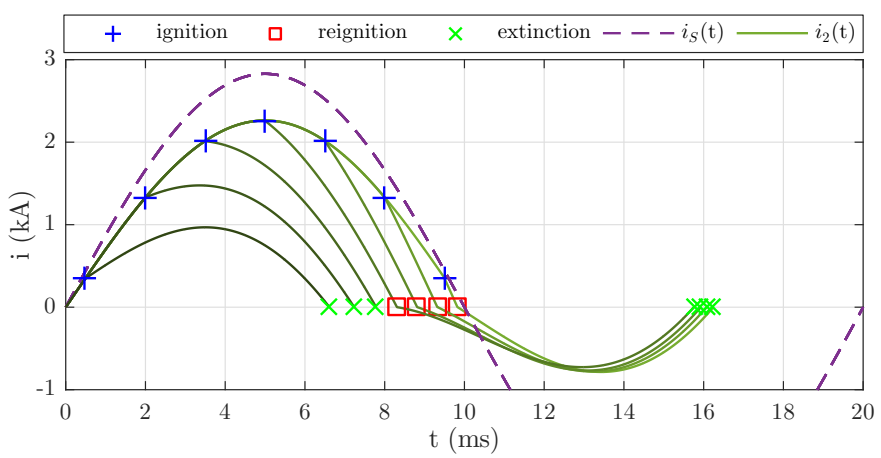

Fig. 7. Simulation of seven ignition times spaced by $1.5 \mathrm{~ms}$ in one half-wave of a total current of $2 \mathrm{kA}$ divided between a $40 \mathrm{~m}$ section of bus in series with the disconnector and a $160 \mathrm{~m}$ parallel section. Blue plus signs indicate ignition of a switching arc, green crosses point to an extinction at current zero, red squares show reignitions. 
TABLE I

COMPARISON OF THE BUS-TRANSFER PROCESSES SHOWN IN FIG. 7 IN TERMS OF IGNITION TIME, ARCING TIME, DISSIPATED ENERGY AND TRANSPORTED CHARGE.

\begin{tabular}{lccccccc}
\hline \hline $\begin{array}{l}\text { ignition } \\
\text { time (ms) }\end{array}$ & 0.5 & 2.0 & 3.5 & 5.0 & 6.5 & 8.0 & 9.5 \\
\hline $\begin{array}{l}\text { arcing } \\
\text { time (ms) }\end{array}$ & 6.1 & 5.2 & 4.3 & 11 & 9.4 & 8.1 & 6.7 \\
$\begin{array}{l}\text { dissipated } \\
\text { energy (J) } \\
\text { transported } \\
\text { charge (C) }\end{array}$ & 64 & 87 & 84 & 130 & 99 & 71 & 52 \\
\hline \hline
\end{tabular}

values of resistance and inductance per unit length are set to $10 \mu \Omega \mathrm{m}^{-1}$ and $200 \mathrm{nH} \mathrm{m}^{-1}$ respectively which are typical values for $420 \mathrm{kV}$ GIS bus [3]. The total current has an amplitude of $2 \mathrm{kA}$ rms, the pre-switching disconnector current therefore $1.6 \mathrm{kA}$. In Fig. 8 the recovery voltage arising from this configuration as well as the exact arc voltage behavior used in the simulation are shown. This example coincides with the bus-transfer test conditions for 245-420 kV GIS according to IEC 62271-102 [1] in terms of pre-switching current as well as recovery voltage. The resulting arcing times, energy dissipated by the arc and total charge transported through the disconnector for each ignition time are given in TABLE I.

From the simulation, it is clear that the time of ignition of the switching arc exhibits a considerable influence on the bus-transfer process itself. Ignitions between $0.5-3.5 \mathrm{~ms}$ with respect to the source current zero exhibit extinction at the first current zero whereas ignitions between $5.0-9.5 \mathrm{~ms}$ after source current zero reignite. This is reflected not only in the arcing time but also in the total energy which is dissipated by the switching arc. An ignition at $5.0 \mathrm{~ms}$ constitutes the worst case with $130 \mathrm{~J}$ which is more than twice the energy expended

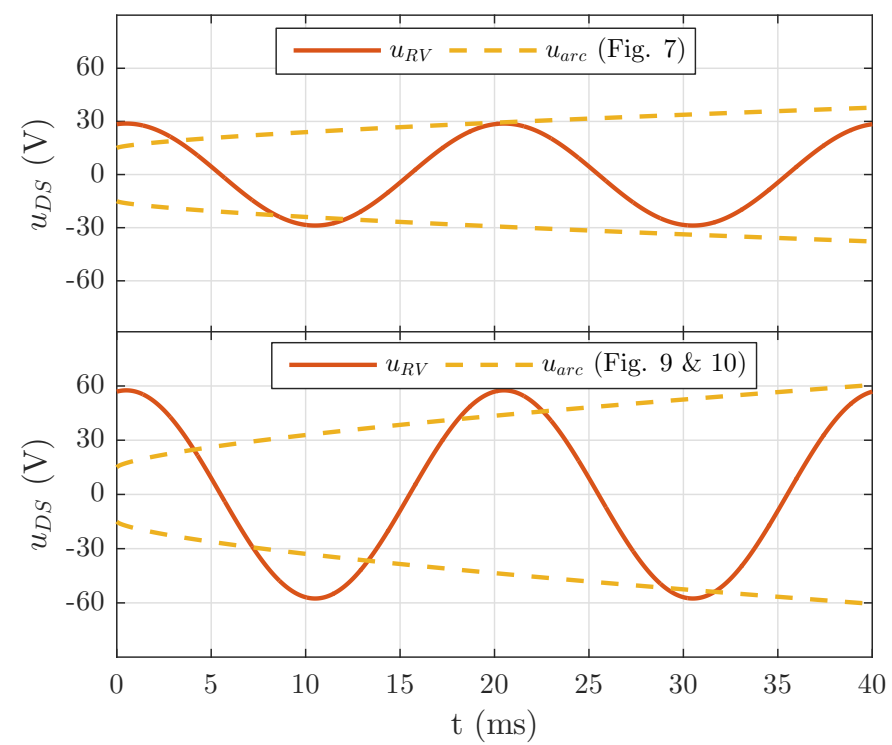

Fig. 8. Arc voltages for an ignition of the switching arc at $0 \mathrm{~ms}$ and recovery voltages used for simulations of the examples. Recovery voltage and arc voltages for Fig. 7 on top, for Fig. 9 and Fig. 10 on bottom. in the best case of ignition at $9.5 \mathrm{~ms}$. Similarly, the charge transported through the arc exhibits a difference over a factor of two between best and worst case. The transported charge can be linked to contact erosion according to Tepper et al. when neglecting the time-dependent heat conduction into the contacts [13]. Therefore, the substantially higher transported charge in the worst case as compared to the best case imply different amounts of mass lost in contact erosion.

Since IEC 62271-102 specifies that 100 bus-transfer tests have to be conducted, the ignition time distribution can become a contributor to the disconnector performance. If tests are conducted favoring ignition times near the beginning or the very end of the half wave of the disconnector current, arcing time and dissipated energy are underrated. Equally, if the specified 100 tests are conducted with ignition around the peak of the disconnector current resulting in long arcing times, unrealistically high stress is put on the contact system. Therefore, it is suggested that repeated tests of bus-transfer switching capability are performed using a uniform distribution of ignition times.

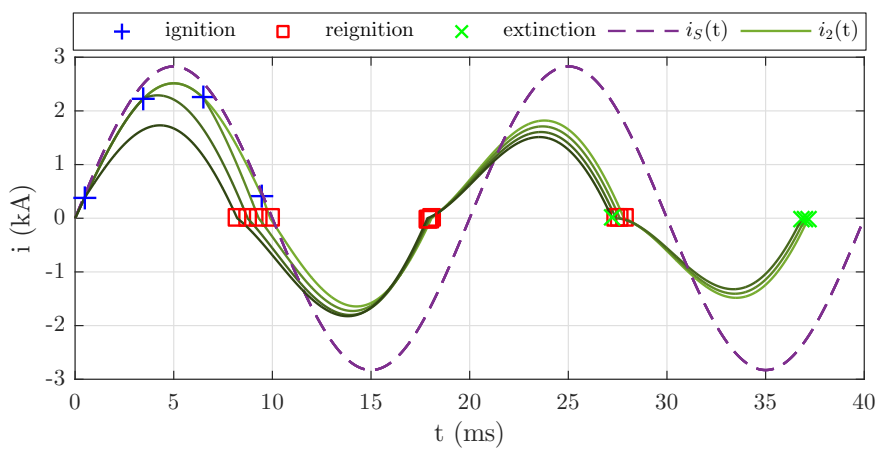

Fig. 9. Simulation of same currents as in Fig. 7 but split between a $320 \mathrm{~m}$ and a $40 \mathrm{~m}$ section of bus.

TABLE II

COMPARISON OF THE BUS-TRANSFER PROCESSES ILLUSTRATED IN FIG. 9 IN TERMS OF IGNITION TIME, ARCING TIME, DISSIPATED ENERGY AND TRANSPORTED CHARGE AT SEVEN DIFFERENT TIME STEPS.

\begin{tabular}{lccccccc}
\hline \hline $\begin{array}{l}\text { ignition } \\
\text { time (ms) }\end{array}$ & 0.5 & 2.0 & 3.5 & 5.0 & 6.5 & 8.0 & 9.5 \\
\hline $\begin{array}{l}\text { arcing } \\
\text { time (ms) }\end{array}$ & 27 & 25 & 33 & 32 & 31 & 29 & 28 \\
$\begin{array}{l}\text { dissipated } \\
\text { energy (J) } \\
\text { transported } \\
\text { charge (C) }\end{array}$ & 620 & 610 & 800 & 800 & 710 & 660 & 620 \\
\hline \hline
\end{tabular}

A second application example for the novel method for predicting limit performance is the simulation of future applications and associated changes of the disconnector. In Fig. 9 the same total current as previously was used but the length of the parallel bus was doubled from $160 \mathrm{~m}$ to $320 \mathrm{~m}$ to exemplify an extension of an existing substation. As a consequence, the pre-switching current distribution changes from $80 \%$ at $1.6 \mathrm{kA}$ through the disconnector to $89 \%$ at $1.8 \mathrm{kA}$ and the recovery voltage doubles in magnitude to $40 \mathrm{~V}$. The effect of these 
changes can be seen in the number of reignitions. The majority of cases only extinguishes after the third reignition. With a range of $620-800 \mathrm{~J}$, the energy dissipated by the switching arcs is significantly higher than in the previous scenario. The same is true for the transported charge which ranges between 29-44 C, one order of magnitude above the previous scenario. In case these charges are above an acceptable limit for electrode erosion, one common solution is to increase the drive speed and therefore the electrode separation speed [14]. When repeating the simulation using an arc voltage function which increases at twice the previous speed, the arcing time decreases as shown in Fig. 10 and TABLE III. Along with the arcing time, the transported charge is reduced to a range of 14-17 C.

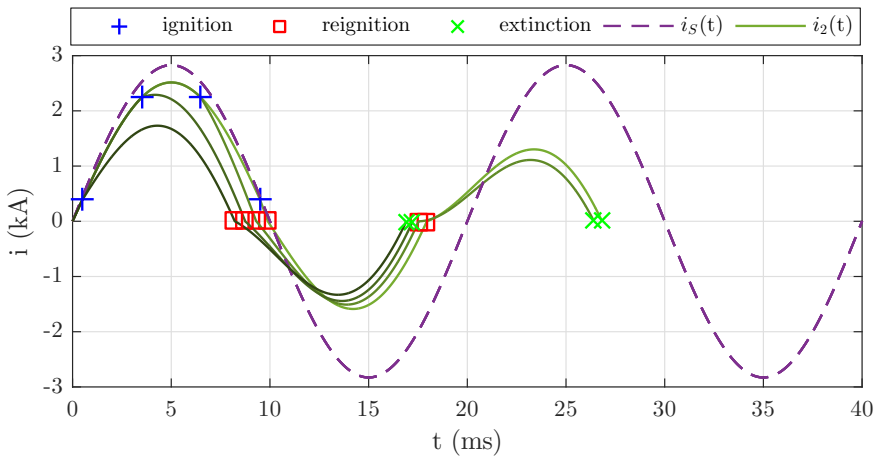

Fig. 10. Simulation of same circuit and current stress as in Fig. 9 interrupted by a disconnector exhibiting an arc voltage increase at twice the previous speed.

TABLE III

COMPARISON OF THE BUS-TRANSFER PROCESSES ILLUSTRATED IN FIG. 10 IN TERMS OF IGNITION TIME, ARCING TIME, DISSIPATED ENERGY AND CHARGE AT SEVEN DIFFERENT TIME STEPS

\begin{tabular}{|c|c|c|c|c|c|c|c|}
\hline $\begin{array}{l}\text { ignition } \\
\text { time (ms) }\end{array}$ & 0.5 & 2.0 & 3.5 & 5.0 & 6.5 & 8.0 & 9.5 \\
\hline $\begin{array}{l}\text { arcing } \\
\text { time (ms) }\end{array}$ & 16 & 15 & 14 & 12 & 20 & 19 & 17 \\
\hline $\begin{array}{l}\text { dissipated } \\
\text { energy }(J)\end{array}$ & 350 & 350 & 330 & 280 & 420 & 380 & 350 \\
\hline $\begin{array}{l}\text { transported } \\
\text { charge }(\mathrm{C})\end{array}$ & 16 & 17 & 16 & 14 & 17 & 15 & 15 \\
\hline
\end{tabular}

The doubling in speed of arc voltage increase has a twofold effect. Primarily, the criterion for reignition of (10) is violated at an earlier time leading to faster extinctions of the current. As a secondary effect, the disconnector current during switching is reduced due to the faster increase of the superimposed arc current according to (6), thus decreasing the energy dissipated by the arc as well as the transported charge and leading to current zeros occurring at earlier times. A comparison of the three simulated scenarios for 100 uniformly distributed ignition times is given in Fig. 11. It is evident from this histogram that changes in disconnector properties as well as substation layout can have significant influence onto bus-transfer arcing times and thus disconnector performance. Using the introduced novel method for predicting limit performance, the

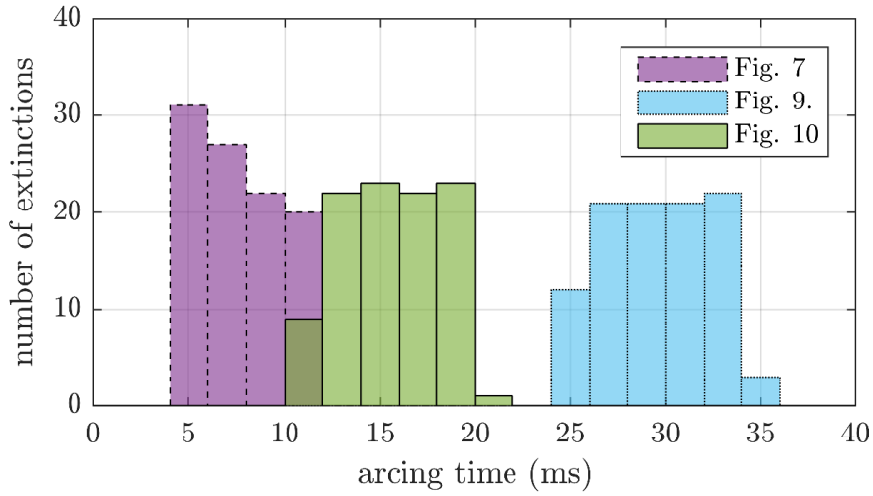

Fig. 11. Classification of the arcing times for 100 uniformly distributed ignition times for each of the three different switching scenarios introduced in Fig. 7, Fig. 9 and Fig. 10.

implications of such changes can be analyzed simulatively with minimal experimental overhead as long as the necessary conditions for the model - such as reasonably short arc length - are not violated.

\section{CONCLUSION}

Based on a large number of bus-transfer tests in a purposebuilt test setup, the switching behavior of a $420 \mathrm{kV} \mathrm{SF}_{6}$ insulated disconnector was investigated. Upon variation of the multiple influencing factors, it was discovered that disconnectorspecific arc voltage in combination with the circuit-defined recovery voltage at the time of each current zero determine if extinction of the switching arc takes place. At current zero, reignition is possible only if the current source is able to drive a current which increases in magnitude faster than the counter current caused by the switching arc. As a criterion this is equivalent to an instantaneous recovery voltage higher than the prospective arc voltage. Therefore, precise simulations of the bus-transfer process are feasible with a minimal knowledge about the source current and bus-transfer loop as well as the arc voltage behavior over time. To illustrate the implications, examples of the influence of ignition time of the switching arc, length of the bus-transfer loop and speed of electrode separation were performed. These simulations highlight how this method for predicting limit performance can be used to asses small changes in testing, application and improvement of such disconnectors minimizing the need for extensive experiment series. When applying this novel method in the development of new disconnectors the need for bus-transfer tests on a laboratory scale is reduced drastically as the prospective arc voltage behavior is the only quantity which has to be determined via high current measurement. The method by which the arc voltage is characterized can be chosen individually, one possibility is using a pulsed DC current source such as the one presented by Walter et al. in [15] which is optimized for large current amplitudes and gradients. 


\section{REFERENCES}

[1] IEC, IEC 62271-102 - Alternating current disconnectors and earthing switches, 1st ed., 2013.

[2] A. Ritter, U. Straumann, U. Riechert, and C. M. Franck, "Investigation of LC-Resonance Driving in Disconnector Bus-Transfer Testing," in Cigré Session 2014, 2014.

[3] Cigré Working Group A3.28, "Switching Phenomena for EHV and UHV Equipment," Cigré Technical Brochure 570, 2014.

[4] A. Ritter, U. Straumann, U. Riechert, and C. M. Franck, "Analysis Method and Implications of Utilizing a Full Bus-Transfer Setup for Testing Disconnectors," in 2015 Cigré SC A3\&B3 Joint Colloquium, 2015.

[5] Y. Yokomizu, T. Matsumura, R. Henmi, and Y. Kito, "Total voltage drops in electrode fall regions of SF6, argon and air arcs in current range from 10 to 20000 A," Journal of Physics D: Applied Physics, vol. 29, no. 5, pp. 1260-1267, may 1996

[6] A. v. Engel and M. Steenbeck, Elektrische Gasentladungen. Berlin, Heidelberg: Springer Berlin Heidelberg, 1932.

[7] A. A. Logachev, P. A. Tenitskiy, and A. V. Vykhodtsev, "Analysis of voltage oscillograms at disconnecting contacts," in 2014 International Symposium on Discharges and Electrical Insulation in Vacuum (ISDEIV). IEEE, sep 2014, pp. 225-228.

[8] P. G. Slade, "The transition from to the metallic phase arc after the rupture of the molten metal bridge for contacts opening in air and vacuum," Electrical Contacts, Proceedings of the Annual Holm Conference on Electrical Contacts, no. 318, pp. 1-8, 2008.

[9] T. W. Dakin, G. Luxa, G. Oppermann, J. Vigreux, G. Wind, and H. Winkelnkemper, "Breakdown of gases in uniform fields. Paschen curves for nitrogen, air and sulfur hexafluoride," Electra, no. 32, pp. 61-82, 1974.

[10] K. Nakanishi, Switching Phenomena in High-Voltage Circuit Breakers, 1991.

[11] S. Franke, R. Methling, D. Uhrlandt, R. Bianchetti, R. Gati, and M. Schwinne, "Temperature determination in copper-dominated freeburning arcs," Journal of Physics D: Applied Physics, vol. 47, no. 1, p. 015202, 2014.

[12] Y. Yokomizu and T. Matsumura, "Radiation power of SF6 arc in current range from 500 to $20000 \mathrm{~A}$ at pressures of 0.1 and $0.4 \mathrm{MPa}$," p. 125203, 2008.

[13] J. Tepper, M. Seeger, T. Votteler, V. Behrens, and T. Honig, "Investigation on Erosion of $\mathrm{Cu} / \mathrm{W}$ Contacts in High-Voltage Circuit Breakers," IEEE Transactions on Components and Packaging Technologies, vol. 29, no. 3, pp. 658-665, sep 2006.

[14] K. Suzuki, H. Mizoguchi, N. Shimokawara, V. Murayama, and S. Yanabu, "Current interruption by disconnecting switch and earthing switch in GIS," Generation, Transmission and Distribution, IEE Proceedings $C$, vol. 131, no. 2, pp. 54-60, 1984.

[15] M. Walter and C. Franck, "Improved Method for Direct Black-Box Arc Parameter Determination and Model Validation," IEEE Transactions on Power Delivery, pp. 1-1, 2014.

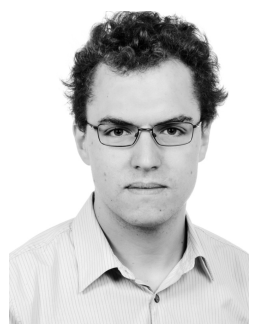

Andreas Ritter (M09) was born in Basel, Switzerland, in 1986. He received the M.Sc. degree in electrical engineering with distinction from ETH Zurich, Switzerland, in 2012. He is currently a scientific assistant at the High Voltage Laboratory at ETH Zurich, researching disconnector and earthing switch operation in future multiterminal HVDC grids.

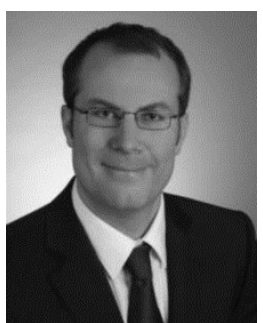

Ueli Straumann (M10SM12) was born in Aarau, Switzerland, in 1975. He received the Ph.D. degree in electrical engineering from ETH Zurich, Zurich, Switzerland, in 2007. Since then, he has been a Lecturer of High Voltage Engineering, ETH Zurich. After being Senior Assistant at the High Voltage Laboratory of ETH Zurich between 2007 and 2012, he became Senior and Principal Engineer at the GIS R\&D of ABB Switzerland Ltd.

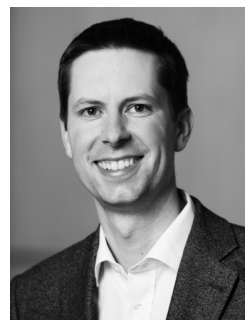

Christian M. Franck (M04 - SM11) received a diploma in physics from the University of Kiel, Germany in 1999 and the Ph.D. degree in physics from the University of Greifswald, Germany in 2003. He was with the Swiss corporate research center of ABB during 2003-2009 as a Scientist and Group Leader for gas circuit breakers and high voltage systems. Currently, he is Associate Professor for High Voltage Technology at the Swiss Federal Institute of Technology (ETH), Zurich, Switzerland. His main research interests are in the area of gaseous insulation systems and equipment for HVDC. 